\title{
Ursodeoxycholic Acid Therapy in Pediatric Primary Sclerosing Cholangitis: Predictors of Gamma Glutamyltransferase Normalization and Favorable Clinical Course
}

Mark Deneau, MD, MS ${ }^{1}$, Emily Perito, $\mathrm{MD}^{2}$, Amanda Ricciuto, MD, $\mathrm{PhD}^{3}$, Nitika Gupta, $\mathrm{MD}^{4}$,

Binita M. Kamath, MD, MBBChir, MRCP ${ }^{3}$, Sirish Palle, $\mathrm{MD}^{5}$, Bernadette Vitola, MD, MPH ${ }^{6}$, Vratislav Smolka, MD ${ }^{7}$, Federica Ferrari, MD, PHD ${ }^{8}$, Achiya Z. Amir, MD ${ }^{9}$, Tamir Miloh, MD ${ }^{10}$, Alexandra Papadopoulou, MD ${ }^{11}$, Parvathi Mohan, MD ${ }^{12}$, Cara Mack, MD ${ }^{13}$, Kaija-Leena Kolho, $\mathrm{MD}^{14}$, Raffaele lorio, MD ${ }^{15}$, Wael El-Matary, MD, MSc ${ }^{16}$, Veena Venkat, MD ${ }^{17}$, Albert Chan, MD ${ }^{18}$, Lawrence Saubermann, MD ${ }^{18}$, Pamela L. Valentino, MD, MSc ${ }^{19}$, Uzma Shah, MBBS ${ }^{20}$, Alexander Miethke, MD ${ }^{21}$, Henry Lin, $\mathrm{MD}^{22}$, and M. K. Jensen, MD, MS ${ }^{1}$

Objective To investigate patient factors predictive of gamma glutamyltransferase (GGT) normalization following ursodeoxycholic acid (UDCA) therapy in children with primary sclerosing cholangitis.

Study design We retrospectively reviewed patient records at 46 centers. We included patients with a baseline serum GGT level $\geq 50 \mathrm{IU} / \mathrm{L}$ at diagnosis of primary sclerosing cholangitis who initiated UDCA therapy within 1 month and continued therapy for at least 1 year. We defined "normalization" as a GGT level $<50 \mathrm{IU} / \mathrm{L}$ without experiencing portal hypertensive or dominant stricture events, liver transplantation, or death during the first year.

Results We identified 263 patients, median age 12.1 years at diagnosis, treated with UDCA at a median dose of $15 \mathrm{mg} / \mathrm{kg} / \mathrm{d}$. Normalization occurred in 46\%. Patients with normalization had a lower prevalence of Crohn's disease, lower total bilirubin level, lower aspartate aminotransferase to platelet ratio index, greater platelet count, and greater serum albumin level at diagnosis. The 5-year survival with native liver was $99 \%$ in those patients who achieved normalization vs $77 \%$ in those who did not.

Conclusions Less than one-half of the patients treated with UDCA have a complete GGT normalization in the first year after diagnosis, but this subset of patients has a favorable 5-year outcome. Normalization is less likely in patients with a Crohn's disease phenotype or a laboratory profile suggestive of more advanced hepatobiliary fibrosis. Patients who do not achieve normalization could reasonably stop UDCA, as they are likely not receiving clinical benefit. Alternative treatments with improved efficacy are needed, particularly for patients with already-advanced disease. (J Pediatr 2019; ם:1-5).

rimary sclerosing cholangitis (PSC) is a rare cholestatic liver disease characterized by progressive destruction of the bile ducts. ${ }^{1}$ Within 10 years of diagnosis, $30 \%$ of children with PSC will require liver transplantation and $50 \%$ of children will develop complications, including biliary stricturing and portal hypertension. ${ }^{2}$ To date, there is no medical intervention proven to prolong patient survival. ${ }^{3}$ PSC is recognized as one the largest unmet needs in hepatology. ${ }^{4}$

Ursodeoxycholic acid (UDCA) is a hydrophilic bile acid with hepatobiliary cytoprotective and immune-modulating effects..$^{5-7}$ The role of UDCA in modifying disease behavior in PSC and prolonging patient survival is controversial. UDCA has not been shown to improve patient survival in adult

$\begin{array}{ll}\text { APRI } & \text { Aspartate aminotransferase to platelet ratio index } \\ \text { AST } & \text { Aspartate aminotransferase } \\ \text { GGT } & \text { Gamma glutamyl transferase } \\ \text { IBD } & \text { Inflammatory bowel disease } \\ \text { PSC } & \text { Primary sclerosing cholangitis } \\ \text { UDCA } & \text { Ursodeoxycholic acid }\end{array}$

From the ${ }^{1}$ Department of Pediatrics, University of Utah, Salt Lake City, UT; ${ }^{2}$ Department of Pediatrics, University of California San Francisco, San Francisco, CA; ${ }^{3}$ Department of Pediatrics, The Hospital for Sick Children, University of Toronto, Toronto, Ontario, Canada; ${ }^{4}$ Department of Pediatrics, Emory University School of Medicine, Atlanta, GA; ${ }^{5}$ Department of Pediatrics, Oklahoma University, Oklahoma City, OK; ${ }^{6}$ Department of Pediatrics, Medical College of Wisconsin, Milwaukee, Wl; ${ }^{7}$ Department of Pediatrics, Palacky University, Olomouc, Czech Republic; ${ }^{8}$ Department of Pediatrics and Pediatric Neuropsychiatry, Sapienza University of Rome, Rome, Italy; 'Division of Gastroenterology, Liver and Nutrition,

The Dana-Dwek Children's Hospital, Tel-Aviv University, Tel Aviv, Israel; ${ }^{10}$ Department of Pediatrics, Texas Children's Hospital, Houston, TX; ${ }^{11}$ First Pediatric Clinic University of Athens, Athens, Greece; ${ }^{12}$ Department of Gastroenterology, Hepatology, and Nutrition, Children's National Medical Center, Washington, DC. ${ }^{13}$ Department of Pediatrics, University of Colorado School of Medicine, Aurora, CO; ${ }^{14}$ Department of Pediatrics, University of Helsinki, Helsinki, Finland; ${ }^{15}$ Department of Pediatrics, University of Naples Federico II, Naples, Italy;

${ }^{16}$ Department of Pediatrics and Child Health, University of Manitoba, Winnipeg, Manitoba, Canada;

${ }^{17}$ Department of Pediatrics, University of Pittsburgh

Medical Center, Pittsburgh, PA; ${ }^{18}$ Department of

Pediatrics, University of Rochester Medical Center

Rochester, NY; ${ }^{19}$ Department of Pediatrics, Yale University School of Medicine, New Haven, CT; ${ }^{20}$ Department of Pediatrics, Harvard University, Boston, MA; ${ }^{21}$ Department of Pediatrics, Cincinnati Children's Hospital Medical Center, Cincinnati, OH; and

${ }^{22}$ Department of Pediatrics, The Children's Hospital of Philadelphia, Philadelphia, PA

Supported by PSC Partners Seeking A Cure, the Primary Children's Hospital Foundation, and the National Center for Advancing Translational Sciences of the National Institutes of Health (KL2TR001065 and 8UL1TR000105 [formerly UL1RR025764]). The content is solely the responsibility of the authors and does not necessarily represent the official views of the National Institutes of Health. M.D. served as a consultant for HighTide Biopharmaceuticals LLC. B.K. is a consultant for Retrophin. T.M. is a consultant, serves on the advisory board, and serves on the speaker board for Alexion. P.M. has received grants from Gilead. The other authors declare no conflicts of interest.

0022-3476/\$ - see front matter. @ 2019 Elsevier Inc. All rights reserved. https://doi.org/10.1016/j.jpeds.2019.01.039 
clinical trials. ${ }^{8-12}$ Given the unclear benefit, as well as a possibility that greater doses of UDCA may cause harm, ${ }^{12}$ the current practice guideline of the American Association of the Study of Liver Diseases recommends against the use of UDCA in adults with PSC. ${ }^{13}$ There are no formal guidelines for the use of UDCA in children with PSC.

A subset of adults normalize serum alkaline phosphatase levels during UDCA therapy and have improved survival compared with those with persistently elevated levels. ${ }^{14-16}$ We showed an analogous effect in children with PSC using gamma glutamyl transferase (GGT) as a biomarker. ${ }^{17}$ Children whose GGT declined to $<50 \mathrm{IU} / \mathrm{L}$ at 1 year after PSC diagnosis had favorable long-term outcomes. Untreated patients who spontaneously normalized serum GGT levels also had good outcomes. The proportion of patients who achieved GGT normalization was greater in those treated with UDCA; however, with an approximate number needed to treat of 4 patients to achieve 1 normalization that would not have occurred on its own. Thus, there appears to be a subgroup of children treated with UDCA who have a favorable response.

UDCA is the most common medication used in children with PSC, with more than $80 \%$ currently receiving long-term therapy. ${ }^{2,18}$ More data are needed to better define which patients may be receiving a clinical benefit from UDCA therapy. There are presently no predictors of which patients will respond. The aim of this study was to identify baseline differences between children with complete vs incomplete biochemical normalization on UDCA.

\section{Methods}

The Pediatric PSC Consortium is an active research collaboration that includes 49 centers in Europe, North America, the Middle East, and Asia. We retrospectively reviewed medical records on all known patients with PSC with disease onset before 18 years of age at each institution, as previously described. ${ }^{2}$ For each patient, we collected data on basic demographics and diagnostic cholangiography and histopathology studies. We collected laboratory data at liver disease diagnosis and approximately 1 year later (within 46-58 weeks after diagnosis). To account for the wide range of normal alkaline phosphatase values in children at various ages due to bone turnover and growth, we normalized all values for age using Mayo Medical Laboratories reference values. ${ }^{19}$

The diagnosis of PSC required elevated hepatobiliary inflammatory markers (GGT or aspartate aminotransferase [AST] or alanine aminotransferase) and/or bilirubin. When characteristic changes on cholangiography were seen, large-duct PSC was diagnosed. When cholangiography was normal but liver histopathology showed typical changes, small-duct PSC was diagnosed. ${ }^{13}$ Cases of sclerosing cholangitis secondary to any other cause were excluded. Autoimmune hepatitis was diagnosed in patients who met the simplified autoimmune hepatitis criteria, which have been validated in children, ${ }^{20}$ based on histopathology, positive autoantibodies, elevated serum globulins, and exclusion of viral hepatitis. We documented the presence and type of associated inflammatory bowel disease (IBD).

We created a retrospective cohort of all patients followed from date of PSC diagnosis to the date of several clinical endpoints: (1) the development of portal hypertensive complications (ascites, hepatic encephalopathy, or esophageal varices with or without bleeding); (2) biliary complications (a clinical picture of biliary obstruction as evidenced by a biliary stricture requiring an intervention in the form of endoscopic or percutaneous stenting, balloon dilation, or drainage); (3) cholangiocarcinoma; (4) liver transplantation; or (5) death from liver disease. Survival free of any of these endpoints was termed event-free survival. Patients were censored at the date of last known follow-up. We excluded patients who presented with portal hypertensive or biliary complications within 3 months of PSC diagnosis from this analysis, because their baseline laboratory studies were likely reflective of the outcomes of interest already being present. Data were abstracted and deidentified at local study sites and reviewed and stored centrally. Study data were collected and managed using REDCap (Research Electronic Data Capture) electronic data capture tools hosted at the University of Utah. ${ }^{21}$

In this analysis, we included patients with baseline serum GGT levels $>50 \mathrm{IU} / \mathrm{L}$ who started UDCA within 1 month of PSC diagnosis and continued taking it for at least 1 year. We defined "normalization" as a decline in GGT to $\leq 50$ IU/L without experiencing clinical endpoints during the first year. The threshold of 50 IU/L for GGT represents 1-2 times the upper limit of normal for most noninfant pediatric age groups at most laboratories and was validated as an endpoint in our previous study. ${ }^{17}$ We compared baseline demographic, phenotypic, and laboratory data between these 2 groups. We assessed differences in the change in laboratory studies between diagnosis and at 1 year. We compared transplant-free survival after 1 year between groups.

The Wilcoxon rank sum and $\chi^{2}$ tests were used to assess differences between groups. We used the Kaplan-Meier method to calculate annual outcome probabilities. The log-rank test assessed survival differences between groups. Logistic regression was used to assess the association between baseline predictor variables and treatment success or failure. Variables with $P$ value $<.1$ in univariate analyses were included in a multivariate model. The model was optimized using variance inflation factor diagnostics, removing any collinear variables. Calculations were done using Stata version 15 (StataCorp, College Station, Texas). All research work was approved by the institutional review board of each participating center.

\section{Results}

We identified 344 patients with a serum GGT level $>50$ IU/L at diagnosis who had laboratory data 1 year later. We 
Table I. Baseline phenotypic and laboratory data

\begin{tabular}{|c|c|c|c|}
\hline & $\begin{array}{c}\text { Normalization } \\
\quad \mathrm{N}=122\end{array}$ & $\begin{array}{l}\text { Non-normalization } \\
\qquad N=141\end{array}$ & $\begin{array}{c}P \\
\text { value }\end{array}$ \\
\hline Age, y & $12.0[7.8-15]$ & $12.4[8.6-15]$ & .416 \\
\hline Sex ( $\%$ male) & $60 \%$ & $60 \%$ & .941 \\
\hline $\begin{array}{l}\text { Ulcerative colitis } \\
\text { (\% with) }\end{array}$ & $74 \%$ & $59 \%$ & .012 \\
\hline $\begin{array}{l}\text { Crohn's disease } \\
\text { (\% with) }\end{array}$ & $10 \%$ & $18 \%$ & .017 \\
\hline No IBD (\% with) & $16 \%$ & $23 \%$ & .203 \\
\hline Autoimmune hepatitis & s $39 \%$ & $39 \%$ & .955 \\
\hline Large-duct phenotype & $e 73 \%$ & $76 \%$ & .586 \\
\hline Hemoglobin, $g / d L$ & $12.4[11.5-13.2]$ & $12.1[11-13.4]$ & .137 \\
\hline Platelet count, $\mathrm{k} / \mu \mathrm{L}$ & 325 [260-438] & 298 [196-375] & $<.001$ \\
\hline INR & $1.1[1-1.2]$ & $1.1[1-1.2]$ & .331 \\
\hline$A L T, U / L$ & $167[87-297]$ & 146 [87-229] & .278 \\
\hline AST, U/L & 125 [71-226] & 140 [78-192] & .697 \\
\hline ALP, U/L & 406 [281-720] & 448 [275-717] & .918 \\
\hline $\operatorname{ALP}(\times \operatorname{ULN})$ & $1.0[0.7-1.6]$ & $1.0[0.6-1.5]$ & .461 \\
\hline GGT, U/L & $290[148-427]$ & 290 [192-465] & .158 \\
\hline Total bilirubin, $\mathrm{mg} / \mathrm{dL}$ & $0.7[0.4-1]$ & $0.9[0.5-1.6]$ & .002 \\
\hline Albumin, $\mathrm{g} / \mathrm{L}$ & $4.1[3.7-4.5]$ & $3.9[3.6-4.3]$ & .008 \\
\hline APRI & $0.9[0.5-1.9]$ & $1.3[0.7-2.3]$ & .023 \\
\hline
\end{tabular}

$A L P$, alkaline phosphatase; $A L T$, alanine aminotransferase; INR, international normalized ratio ULN, upper limit of normal.

Values are represented as median [interquartile range]. Values in bold are statistically significant.

excluded 63 patients who did not receive UDCA treatment and 18 patients with clinical complications at or within 3 months after diagnosis. The remaining 263 patients $(60 \%$ male, median age 12.1 years old, median GGT 290 [IQR 163-431]) were followed for a median of 5.4 years with a total of 1736 person-years of observation. The median UDCA dose administered was $15 \mathrm{mg} / \mathrm{kg} / \mathrm{d}$ (IQR 15-19).

Serum GGT normalization occurred in 46\% (122/263) of patients. In the remainder, non-normalization occurred in $16 \%(23 / 141)$ due to occurrence of clinical events and in $84 \%$ due to persistently elevated GGT at 1 year. Patients with normalization vs non-normalization had similar PSC phenotypes. At diagnosis, however, the response group had

\begin{tabular}{|c|c|c|c|}
\hline & $\begin{array}{c}\text { Normalization } \\
n=122\end{array}$ & $\begin{array}{c}\text { Non-normalization } \\
n=141\end{array}$ & $\begin{array}{c}P \\
\text { value }\end{array}$ \\
\hline $\begin{array}{l}\text { Hemoglobin, } \\
\text { g/dL }\end{array}$ & $+0.8[-0.4$ to +1.6$]$ & $+0.6[-0.4$ to +1.5$]$ & .382 \\
\hline $\begin{array}{l}\text { Platelet count, } \\
\quad \mathrm{K} / \mu \mathrm{L}\end{array}$ & $-34[-114$ to +5$]$ & $-32[-87$ to +22$]$ & .132 \\
\hline INR & $0[-0.1$ to +0.1$]$ & $0[-0.1$ to +0.2$]$ & .600 \\
\hline$A L T, U / L$ & $-141[-266$ to -59$]$ & $-70[-165$ to +3$]$ & $<.001$ \\
\hline AST, U/L & $-92[-197$ to -40$]$ & $-62[-151$ to -7$]$ & $<.001$ \\
\hline$A L P, U / L$ & $-278[-411$ to +2$]$ & $-129[-618$ to +80$]$ & $<.001$ \\
\hline $\operatorname{ALP}(\times \operatorname{ULN})$ & $-0.6[-1.3$ to -0.2$]$ & $-0.3[-0.9$ to 0$]$ & $<.001$ \\
\hline GGT, U/L & $-258[-406$ to -127$]$ & $-142[-271$ to -42$]$ & $<.001$ \\
\hline $\begin{array}{l}\text { Total bilirubin, } \\
\mathrm{mg} / \mathrm{dL}\end{array}$ & $-0[-0.4$ to +0.2$]$ & $+0.1[-0.7$ to +0.2$]$ & .567 \\
\hline Albumin, $g / L$ & $+0.2[-0.1$ to +0.6$]$ & $+0.1[-0.3$ to +0.4$]$ & .038 \\
\hline APRI & $-0.7[-1.6$ to -0.2$]$ & $-0.4[-1.3$ to 0$]$ & .023 \\
\hline
\end{tabular}

Values are represented as median [interquartile range]. Values in bold are statistically significant.

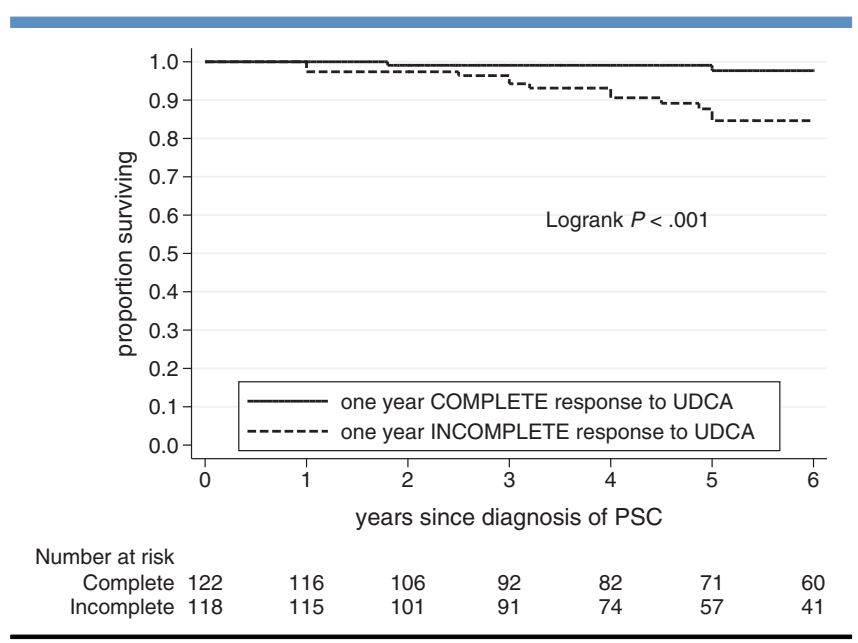

Figure 1. Long-term survival with native liver in patients treated with UDCA.

a lower prevalence of Crohn's disease, lower total bilirubin, lower aspartate aminotransferase to platelet ratio index (APRI), greater platelet count, and greater serum albumin (Table I). In addition to the primary endpoint of GGT reduction, the normalization group experienced larger improvements in alanine aminotransferase, AST, alkaline phosphatase, and APRI compared with those with non-normalization in the year after PSC diagnosis (Table II).

The presence or absence of a Crohn's disease IBD phenotype, as well as baseline platelet count, total bilirubin, and albumin at diagnosis showed significant univariate correlation with treatment response (Table III; available at www.jpeds.com). After regression diagnostics including all variables with $P<.10$ in univariate analysis, hemoglobin was removed for excessive covariance. In the final multivariate model, a Crohn's phenotype decreased the odds of normalization. Increased platelet count and serum albumin significantly increased the odds of a normalization (Table IV; available at www.jpeds.com).

Long-term survival with native liver after 1 year was better in the normalization group than the incomplete response group (Figure 1). Following the first year of UDCA treatment, the annual event rate (deaths or liver transplants per group per year) was $0.7 \%$ year in the normalization group vs $4.3 \% /$ year in the non-normalization group, $\log$-rank $P<.001$. The 5-year survival with native liver was $99 \%$ (95\% CI 94-100) in those with normalization, compared with 77\% (95\% CI 68-84) in those who did not achieve normalization, $P<.001$.

Among those who did not normalize GGT on UDCA who did not experience an adverse clinical event within the first year, event-free survival from years 1 to 6 was different based on the percentage GGT reduction achieved by 1 year (Figure 2). There was a trend toward the best event-free survival for patients with the largest relative GGT reductions between diagnosis and year 1 . Those with a $>75 \%$ reduction in GGT, compared with a $<25 \%$ reduction 


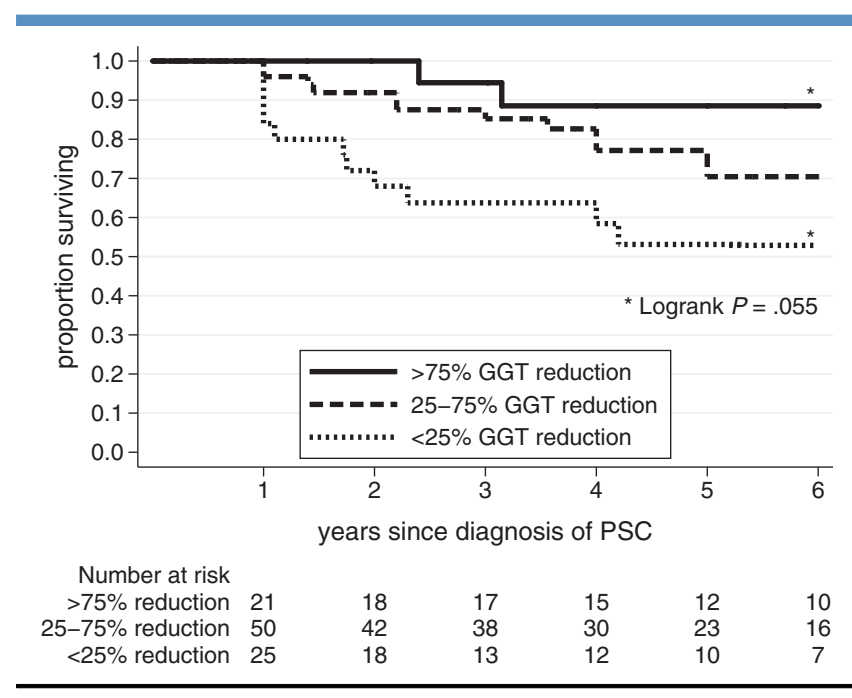

Figure 2. Event-free survival after 1 year in patients with incomplete biochemical response to UDCA, stratified by percentage reduction in GGT from baseline.

in GGT, had an $88 \%$ vs $52 \%$ event-free survival over the next 5 years, $P=.055$.

\section{Discussion}

Although only a minority of children experience normalization of their serum GGT level in response to UDCA therapy, those who do show a decreased likelihood of liver transplant or death during follow-up. We identified clinically relevant patient factors at baseline associated with non-normalization on UDCA treatment, including a Crohn's disease IBD phenotype, relative thrombocytopenia, hypoalbuminemia, or hyperbilirubinemia, and increased APRI, a surrogate marker of fibrosis.

The $46 \%$ normalization rate observed in this study is similar to a $41 \%$ biochemical response seen in an adult prospective trial of UDCA. ${ }^{15}$ In our pediatric cohort, outcomes were best in those with GGT $<50 \mathrm{IU} / \mathrm{L}$ at 1 year after diagnosis. Even among those with GGT $\geq 50 \mathrm{IU} / \mathrm{L}$ at 1 year, outcomes were better with progressively larger reductions in GGT between diagnosis and 1 year. Those who had a reduction in GGT of $>75 \%$ had nearly the same long-term survival as those with GGT $<50$ IU/L at 1 year. These data further support the utility of GGT reduction as a candidate surrogate endpoint for future clinical trials. Clinically, children with PSC on long-term UDCA who have not had a $>75 \%$ reduction in GGT or complete normalization of GGT can stop the medicine, as they are unlikely to be receiving a clinical benefit and could minimize unnecessary drug exposure.

Of note, we do not know how many of the patients treated with UDCA would have had spontaneous GGT normalization and no adverse liver events without UDCA therapy. We previously showed that nearly one-third of children who are UDCA-naive have spontaneous GGT normalization by 1 year. These untreated patients' long-term survival outcomes are indistinguishable from patients treated with UDCA with GGT normalization. ${ }^{17} \mathrm{~A}$ larger proportion of patients treated with UDCA achieved GGT normalization, however. The number of patients with PSC who would need to be treated with UDCA to achieve 1 case of GGT normalization that would not have occurred spontaneously without treatment was approximately 4 (number needed to treat $=4$ ).

A recent clinical trial evaluated the impact of UDCA withdrawal from children with PSC who had been on chronic therapy with normal liver biochemistries. Upon complete withdrawal of the medication for 12 weeks, one-third of patients maintained serum GGT levels $<29$ IU/L. ${ }^{22}$ Whether the one-third of patients who may have spontaneously normalized GGT and the one-third of patients who maintained a normal GGT on complete withdrawal of UDCA represent the same group is speculative, but it is likely that a large proportion of children on chronic UDCA are receiving no clinical benefit. More data on the natural history of GGT in patients exposed and not exposed to UDCA within the first 3-6 months after diagnosis are needed to determine the optimal length of a trial of UDCA therapy.

Liver disease phenotype was not associated with normalization: patients with large-and small-duct disease had equivalent normalization rates, and the presence of autoimmune hepatitis overlap features did not affect outcome. Similarly, no phenotypic differences were observed in UDCA complete vs incomplete biochemical changes in an adult randomized-controlled trial. ${ }^{10}$ Biochemical differences between groups at baseline suggest that patients with more extensive hepatobiliary fibrosis were less likely to achieve normalization on UDCA. There was a consistent trend across several markers: decreased serum albumin and platelet count, and increased APRI and bilirubin in non-normalizers. Length of time with PSC before diagnosis is likely an important, but ultimately unmeasurable, factor. Patient age at diagnosis was similar in response and incomplete response groups. We speculate that there may be additional subphenotypes of PSC that are more slowly progressive and more responsive to therapy, perhaps based on microbiome or metabolome profiles.

Crohn's disease had a negative impact on the probability of normalization. This was in contrast to the association between Crohn's disease and a favorable PSC prognosis in general. The presence of IBD overall (either ulcerative colitis or a Crohn's disease phenotype) is a favorable predictor of outcome in pediatric PSC, ${ }^{2}$ and a Crohn's disease phenotype was a favorable predictor in adult PSC. ${ }^{23}$ Patients with Crohn's disease may be more likely to receive immunomodulatory or biologic medicines than patients with ulcerative colitis or those with no IBD, although such medicines have not shown a survival detriment or benefit in PSC. ${ }^{24,25}$ Differences in the microbiome of patients with Crohn's disease may not favor useful, disease-modifying metabolism of UDCA in some patients. Indeed, patients 
with PSC-Crohn's, PSC-ulcerative colitis, and PSC with no IBD each have distinct microbiota signatures, ${ }^{26}$ with underrepresentation of Butyricicoccus in PSC-Crohn's compared with the others. The microbiome may impact for PSC progression itself and, metabolism of and potential responsiveness to, UDCA. Additional research is needed to more fully characterize the relative effectiveness of medical therapies for PSC in patients with different microbiome profiles and across the spectrum of objectively staged hepatobiliary fibrosis and duct stricturing.

A strength of this study was its large size and inclusion of patients from a diverse mix of secondary and tertiary referral centers. There are weaknesses to this study. The retrospective design prevented a standardized diagnostic and therapeutic algorithm, and misclassification bias may be present. We did not have data on compliance with prescribed UDCA, which may have been poor especially in teenage patients. GGT and general biochemistry was only available at the time of diagnosis and 1 year later. Data at earlier time points to report on rapidity of GGT improvement were not available. Similarly, longer-term data are needed to define the durability of GGT normalization on and off chronic UDCA therapy. Future studies should control for the severity of intestinal disease and concomitant immunosuppression for IBD and autoimmune hepatitis, which we were unable to do here. Finally, objective markers of disease severity and response are needed, including performing liver biopsy and cholangiography before and during therapy. These data are part of a larger, more extensive data collection that is currently underway within the Pediatric PSC Consortium to address these shortfalls.

Submitted for publication Nov 16, 2018; last revision received Jan 17, 2019; accepted Jan 18, 2019.

Reprint requests: Mark Deneau, MD, MS, Associate Professor of Pediatrics, Division of Gastroenterology, Hepatology and Nutrition, University of Utah, Intermountain Healthcare / Primary Children's Hospital, 81 N. Mario Capecchi Dr, Salt Lake City, UT 84113. E-mail: mark.deneau@hsc.utah.edu

\section{References}

1. Hirschfield GM, Karlsen TH, Lindor KD, Adams DH. Primary sclerosing cholangitis. Lancet 2013;382:1587-99.

2. Deneau MR, El-Matary W, Valentino PL, Abdou R, Alqoaer K, Amin M, et al. The natural history of primary sclerosing cholangitis in 781 children: a multicenter, international collaboration. Hepatology 2017;66:518-27.

3. Karlsen TH, Folseraas T, Thorburn D, Vesterhus M. Primary sclerosing cholangitis-a comprehensive review. J Hepatol 2017;67:1298-323.

4. Dyson JK, Webb G, Hirschfield GM, Lohse A, Beuers U, Lindor K, et al. Unmet clinical need in autoimmune liver diseases. J Hepatol 2015;62: 208-18.

5. Galle PR, Theilmann L, Raedsch R, Otto G, Stiehl A. Ursodeoxycholate reduces hepatotoxicity of bile salts in primary human hepatocytes. Hepatology 1990;12:486-91.

6. Stiehl A, Rudolph G, Raedsch R, Möller B, Hopf U, Lotterer E, et al. Ursodeoxycholic acid-induced changes of plasma and urinary bile acids in patients with primary biliary cirrhosis. Hepatology 1990;12:492-7.

7. Calmus Y, Gane P, Rouger P, Poupon R. Hepatic expression of class I and class II major histocompatibility complex molecules in primary biliary cirrhosis: effect of ursodeoxycholic acid. Hepatology 1990;11: 12-5.
8. Lindor KD. Ursodiol for primary sclerosing cholangitis. Mayo Primary Sclerosing Cholangitis-Ursodeoxycholic Acid Study Group. N Engl J Med 1997;336:691-5.

9. Cullen SN, Rust C, Fleming K, Edwards C, Beuers U, Chapman RW. High dose ursodeoxycholic acid for the treatment of primary sclerosing cholangitis is safe and effective. J Hepatol 2008;48:792-800.

10. Olsson R, Boberg KM, de Muckadell OS, Lindgren S, Hultcrantz R, Folvik G, et al. High-dose ursodeoxycholic acid in primary sclerosing cholangitis: a 5-year multicenter, randomized, controlled study. Gastroenterology 2005;129:1464-72.

11. Harnois DM, Angulo P, Jorgensen RA, Larusso NF, Lindor KD. High-dose ursodeoxycholic acid as a therapy for patients with primary sclerosing cholangitis. Am J Gastroenterol 2001;96:1558-62.

12. Lindor KD, Kowdley KV, Luketic VA, Harrison ME, McCashland T, Befeler AS, et al. High-dose ursodeoxycholic acid for the treatment of primary sclerosing cholangitis. Hepatology 2009;50:808-14.

13. Chapman R, Fevery J, Kalloo A, Nagorney DM, Boberg KM, Shneider B, et al. Diagnosis and management of primary sclerosing cholangitis. Hepatology 2010;51:660-78.

14. Al Mamari S, Djordjevic J, Halliday JS, Chapman RW. Improvement of serum alkaline phosphatase to $<1.5$ upper limit of normal predicts better outcome and reduced risk of cholangiocarcinoma in primary sclerosing cholangitis. J Hepatol 2013;58:329-34.

15. Lindstrom L, Hultcrantz R, Boberg KM, Friis-Liby I, Bergquist A. Association between reduced levels of alkaline phosphatase and survival times of patients with primary sclerosing cholangitis. Clin Gastroenterol Hepatol 2013;11:841-6.

16. Stanich PP, Bjornsson E, Gossard AA, Enders F, Jorgensen R, Lindor KD. Alkaline phosphatase normalization is associated with better prognosis in primary sclerosing cholangitis. Dig Liver Dis 2011;43:309-13.

17. Deneau MR, Mack C, Abdou R, Amin M, Amir A, Auth M, et al. Gamma glutamyltransferase reduction is associated with favorable outcomes in pediatric primary sclerosing cholangitis. Hepatol Commun 2018;2: 1369-78.

18. Valentino PL, Wiggins S, Harney S, Raza R, Lee CK, Jonas MM. The natural history of primary sclerosing cholangitis in children: a large single-center longitudinal cohort study. J Pediatr Gastroenterol Nutr 2016;63:603-9.

19. Mayo Clinic Laboratories. Alkaline Phosphatase, Total and Isozymes, Serum: Clinical and Interpretive, http://www.mayomedicallaboratories. com/test-catalog/Clinical+and+Interpretive/89503. Accessed August 18, 2016.

20. Mileti E, Rosenthal P, Peters MG. Validation and modification of simplified diagnostic criteria for autoimmune hepatitis in children. Clin Gastroenterol Hepatol 2012;10:417-21.e1-2.

21. Harris PA, Taylor R, Thielke R, Payne J, Gonzalez N, Conde JG. Research electronic data capture (REDCap) — a metadata-driven methodology and workflow process for providing translational research informatics support. J Biomed Inform 2009;42:377-81.

22. Black DMC, Kerkar N, Miloh T, Sundaram S, Anand R, Gupta A, et al. Initial results of the WUPPSC Study-Prospective Multicenter Withdrawal of Ursodeoxycholic Acid in Pediatric Primary Sclerosing Cholangitis [Abstract]. Gastroenterology 2018;154(6 S1):S1209.

23. Weismuller TJ, Trivedi PJ, Bergquist A, Imam M, Lenzen $H$, Ponsioen CY, et al. Patient age, sex, and inflammatory bowel disease phenotype associate with course of primary sclerosing cholangitis. Gastroenterology 2017;152:1975-84.e8.

24. Tse CS, Loftus EV Jr, Raffals LE, Gossard AA, Lightner AL. Effects of vedolizumab, adalimumab and infliximab on biliary inflammation in individuals with primary sclerosing cholangitis and inflammatory bowel disease. Aliment Pharmacol Ther 2018;48:190-5.

25. Peng X, Luo X, Hou JY, Wu SY, Li LZ, Zheng MH, et al. Immunosuppressive agents for the treatment of primary sclerosing cholangitis: a systematic review and meta-analysis. Dig Dis 2017;35: 478-85.

26. Sabino J, Vieira-Silva S, Machiels K, Joossens M, Falony G, Ballet V, et al. Primary sclerosing cholangitis is characterised by intestinal dysbiosis independent from IBD. Gut 2016;65:1681-9. 
Table III. Univariate regression analysis of baseline predictors of normalization on UDCA

\begin{tabular}{lcr} 
& Univariate $\mathbf{~ O R}$ & P value \\
\hline Age & 0.97 & .328 \\
Sex & 0.98 & .941 \\
UC & 1.35 & .235 \\
Crohn's & $\mathbf{0 . 4 1}$ &. $\mathbf{0 2 6}$ \\
No IBD & 0.57 & .094 \\
AlH & 1.01 & .955 \\
Large-duct phenotype & 0.84 & .586 \\
Hemoglobin & 1.15 & .071 \\
Platelet count & $\mathbf{1 . 4 3}$ & $<.001$ \\
INR & 0.82 & .795 \\
ALT & 1.00 & .660 \\
AST & 1.00 & .710 \\
ALP & 0.99 & .943 \\
ALP & 1.01 & .906 \\
GGT & 0.99 & .092 \\
Total bilirubin & $\mathbf{0 . 8 7}$ &. $\mathbf{0 0 5}$ \\
Albumin & $\mathbf{1 . 8 4}$ & .008 \\
APRI & 0.95 & .155 \\
\hline
\end{tabular}

$A l H$, autoimmune hepatitis; $A L P$, alkaline phosphatase; $A L T$, alanine aminotransferase; $I N R$, international normalized ratio; $U C$, ulcerative colitis.

Values in bold are statistically significant.
Table IV. Multivariate model of baseline predictors of normalization on UDCA

\begin{tabular}{|c|c|c|}
\hline & Multivariate $\mathbf{O R}$ & $P$ value \\
\hline Crohn's (vs UC or no IBD) & 0.33 & .012 \\
\hline Platelet count (per $100000 \mathrm{k} / \mu \mathrm{L}$ ) & 1.55 & $<.001$ \\
\hline GGT (per U/L) & 0.99 & .119 \\
\hline Total bilirubin (per $\mathrm{mg} / \mathrm{dL}$ ) & 0.88 & .167 \\
\hline Albumin (per g/dL) & 1.98 & .009 \\
\hline
\end{tabular}

Values in bold are statistically significant. 